\title{
SHAPING OF NOVA SHELLS BY BINARY MOTION
}

\author{
H. M. LLOYD, T. J. O'BRIEN, M. F. BODE \\ Astrophysics Group, Liverpool John Moores University, Byrom \\ Street, Liverpool L3 $3 A F$, UK
}

\begin{abstract}
Several years after outburst, extended optical emission can be seen around many classical novae. Common features include shells, polar 'blobs', equatorial rings and, at least in the case of DQ Her (1934), tropical rings above and below the 'equator'. We present hydrodynamic calculations of the dynamical effects of the underlying binary system on the material ejected during outburst, and show that many of the features observed in the optical remnants of novae can be reproduced. Polar blobs, banded shells and spherical shells can all be obtained in the model, depending on the speed class of the nova.
\end{abstract}

There is strong evidence to support the fact that the velocity of ejection in novae increases with time (see e.g. Friedjung 1994) which will naturally lead to a situation in which fast moving ejecta will catch up with slower moving material ejected earlier in the outburst, sweeping it up into a shell and shock-heating it to X-ray temperatures. A spherically symmetric calculation of this 'interacting winds' model was performed by O'Brien, Lloyd \& Bode (1994) in order to model the X-ray emission from V838 Her. Here we extend the model to axisymmetry to allow for the effects of the binary system, which will deposit energy and angular momentum into the flow via a gravitational drag force. The hydrodynamic code uses a spherical polar grid, and the $\phi$ velocity is allowed to be non-zero (the '2.5-D' approximation). Previous 2-D calculations of these effects have only been performed for slow novae (Livio et al. 1990).

The mass-loss is assumed to take place in a wind, with a velocity which increases with time. Orbital energy and angular momentum are added into the flow at the orbital radius of the secondary star. Four runs of the code were performed with velocities appropriate for slow (Run 1), moderately fast (Runs 2 and 3) and fast (Run 4) novae. Fig. 1 shows model images obtained from the four runs (at times after the acceleration of the wind

A. Evans and J. H. Wood (eds.), Cataclysmic Variables and Related Objects, 313-314.

(1) 1996 Kluwer Academic Publishers. Printed in the Netherlands. 


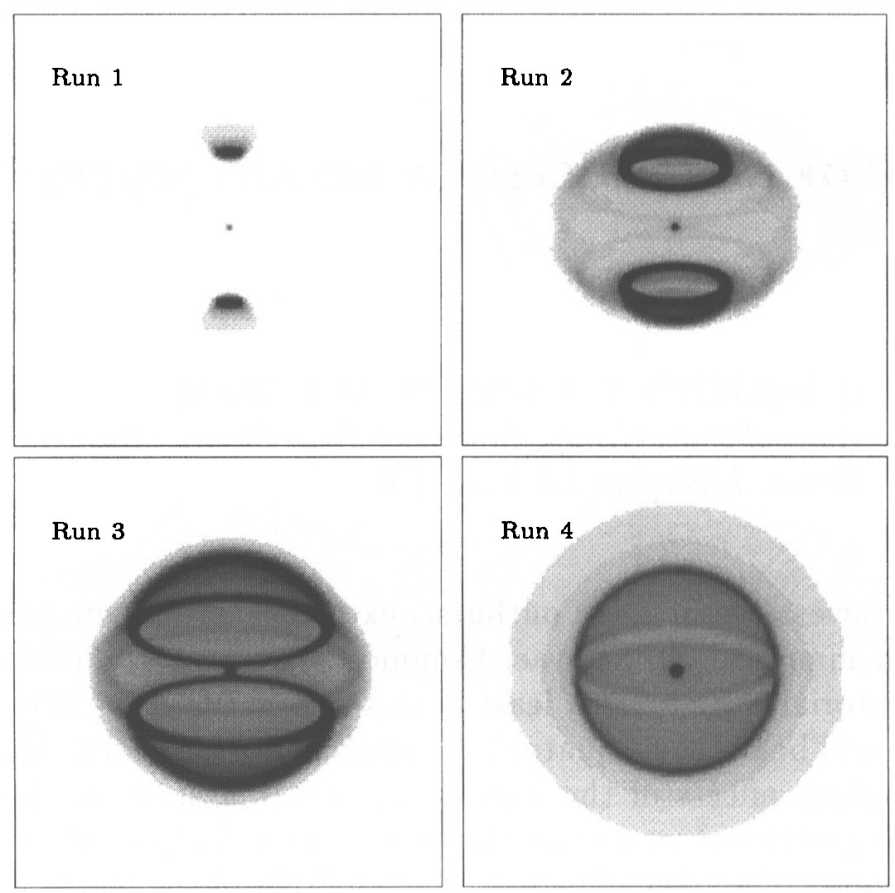

Figure 1. Synthetic images obtained from the hydrodynamic models.

is complete) by taking the emission coefficient to be proportional to the square of the gas density. Run 3 shows a banded shell similar to that seen in DQ Her (Slavin, O'Brien \& Dunlop 1995) whereas Run 2 shows polar rings. Both these structures are formed via the mechanism described by Lloyd, O'Brien \& Kahn (1995) in their model for the nebula of SN1987a. The slow wind is shaped into a double cone structure which is then swept up by the fast wind to give a pair of rings. The effect of the drag force is greater for lower velocities and, in the case of Run 1, the cone is forced to collapse onto the symmetry axis and a bipolar structure is obtained (such as that seen in HR Del, Slavin, O'Brien \& Dunlop 1994). The mass loss in the fast nova model (Run 4) is relatively unaffected by the drag force and results in an almost spherical shell.

\section{References}

Friedjung, M., 1994, Mem. S. A. It., 65, 1

Livio, M., Shankar, A., Burkert, A., Truran, J. W., 1990, Ap. J., 356, 250

Lloyd, H. M., O'Brien, T. J., Kahn, F. D., 1995, MNRAS, 273, L19

O'Brien, T. J., Lloyd, H. M., Bode, M. F., 1994, MNRAS, 271, 155

Slavin, A. J., O'Brien, T. J., Dunlop, J. S., 1994, MNRAS, 266, L55

Slavin, A. J., O'Brien, T. J., Dunlop, J. S., 1995, MNRAS, 276, 353 